\title{
THE DEGREE OF ILL-POSEDNESS OF COMPOSITE LINEAR ILL-POSED PROBLEMS WITH FOCUS ON THE IMPACT OF THE NON-COMPACT HAUSDORFF MOMENT OPERATOR*
}

\author{
${\text { BERND HOFMANN }{ }^{\dagger} \text { AND PETER MATHÉ } \ddagger}^{\ddagger}$
}

\begin{abstract}
We consider compact composite linear operators in Hilbert space, where the composition is given by some compact operator followed by some non-compact one possessing a non-closed range. Focus is on the impact of the non-compact factor on the overall behavior of the decay rates of the singular values of the composition. Specifically, the composition of the compact integration operator with the non-compact Hausdorff moment operator is considered. We show that the singular values of the composite operator decay faster than those of the integration operator, providing a first example of this kind. However, there is a gap between available lower bounds for the decay rate and the obtained result. Therefore we conclude with a discussion.
\end{abstract}

Key words. Hausdorff moment problem, linear inverse problem, degree of ill-posedness, composite operator, conditional stability

AMS subject classifications. 47A52, 47B06, 65J20, 44A60

1. Introduction. We consider the following composite linear ill-posed operator equation $A x=y$ with

$$
A: X \stackrel{D}{\longrightarrow} Z \stackrel{B}{\longrightarrow} Y,
$$

where $A=B \circ D: X \rightarrow Y$ denotes the compact linear operator with infinite-dimensional range $\mathcal{R}(A)$. This forward operator $A$ is a composition of a compact linear operator $D: X \rightarrow$ $Z$ with infinite-dimensional range $\mathcal{R}(D)$ and a bounded non-compact linear operator $B: Z \rightarrow$ $Y$ with non-closed range $\mathcal{R}(B) \neq \overline{\mathcal{R}}(B)^{Y}$. Here $X, Y$, and $Z$ denote three infinitedimensional separable real Hilbert spaces. In the nomenclature of Nashed [16], the inner problem is a linear operator equation

$$
D x=z,
$$

which is ill-posed of type II due to the compactness of $D$, whereas the outer problem

$$
B z=y
$$

is ill-posed of type $\mathrm{I}$, since $B$ is non-compact.

Operator equations with non-compact operators possessing a non-closed range are often assumed to be less ill-posed (ill-posedness of type I), and we refer to Nashed [16, p. 55] who states that " $\ldots$ an equation involving a bounded non-compact operator with non-closed range is 'less' ill-posed than an equation with a compact operator with infinite-dimensional range." For compact operator equations it is common to measure the degree of ill-posedness in terms of the decay rate of the singular values, and the above composite operator (1.1) is of this type despite the non-compact factor $B$.

In our subsequent analysis we will mainly analyze and compare the following cases, which are of the above type and seemingly should have similar properties. The compact factor $D$ is given either

* Received November 11, 2021. Accepted December 9, 2021. Published online on March 2, 2022. Recommended by Stefan Kindermann.

${ }^{\dagger}$ Faculty of Mathematics, Chemnitz University of Technology, 09107 Chemnitz, Germany (hofmannb@mathematik.tu-chemnitz.de).

${ }^{\ddagger}$ Weierstraß Institute for Applied Analysis and Stochastics, Mohrenstraße 39, 10117 Berlin, Germany (mathe@wias-berlin.de). 
- as the simple integration operator

$$
[J x](s):=\int_{0}^{s} x(t) \mathrm{d} t \quad(0 \leq s \leq 1)
$$

mapping in $L^{2}(0,1)$, or

- as the natural (compact) embedding

$$
\mathcal{E}^{(k)}: H^{k}(0,1) \hookrightarrow L^{2}(0,1)
$$

from the Sobolev space $H^{k}(0,1)$ of order $k \in \mathbb{N}$ to $L^{2}(0,1)$.

This will be composed with $B$ being either

- a bounded linear multiplication operator

$$
\left[B^{(\mathrm{M})} x\right](t):=m(t) x(t) \quad(0 \leq t \leq 1)
$$

with a multiplier function $m \in L^{\infty}(0,1)$ possessing essential zeros, or - the Hausdorff moment operator $B^{(\mathrm{H})}: Z=L^{2}(0,1) \rightarrow Y=\ell^{2}$ defined as

$$
\left[B^{(\mathrm{H})} z\right]_{j}:=\int_{0}^{1} t^{j-1} z(t) \mathrm{d} t \quad(j=1,2, \ldots) .
$$

The inner operators (1.4) and (1.5) are known to be compact, even Hilbert-Schmidt, and the decay rates of their singular values $\sigma_{i}(J)$ and $\sigma_{i}\left(\mathcal{E}^{(k)}\right)$ to zero are available. Both the above outer operators (1.6) and (1.7) are known to be non-compact with non-closed range.

The composition $B^{(\mathrm{M})} \circ J$ was studied in $[6,11,12,24]$. Recent studies of the Hausdorff moment problem, which goes back to Hausdorff's paper [8], have been presented in [7]. In particular, we refer to [7, Theorem 1 and Proposition 13], which yield assertions for the composition of type $B^{(\mathrm{H})} \circ \mathcal{E}^{(k)}$.

The question that we are going to address is the following. What is, in terms of the decay of the singular values $\sigma_{i}(B \circ D)$ of the composite operator $B \circ D$ from (1.1), the impact of the non-compact outer operator $B$ ?

In the case of $B:=B^{(\mathrm{M})}$ and $D:=J$, results are known. For several classes of multiplier functions $m$, including $m(t)=t^{\theta}$ for all $\theta>0$, it was seen that the singular values of the composite operator $A$ obey the equivalence ${ }^{1}$

$$
\sigma_{i}(A)=\sigma_{i}\left(B^{(\mathrm{M})} \circ J\right) \asymp \sigma_{i}(J) \asymp \frac{1}{i} \quad \text { as } i \rightarrow \infty,
$$

which means that $B^{(\mathrm{M})}$ does not "destroy" the degree of ill-posedness of $J$ by composition.

REMARK 1.1. The right-hand inequalities $\sigma_{i}(B \circ J) \leq \bar{c} \sigma_{i}(J)$, for example, required in (1.8), are trivially satisfied if $B$ is bounded. We have $\sigma_{i}\left(B^{(\mathrm{M})} \circ J\right) \leq C \sigma_{i}(J)$ with $C:=$ $\left\|B^{(\mathrm{M})}\right\|_{L^{2}(0,1) \rightarrow L^{2}(0,1)}$. Clearly, the same reasoning applies to the composite operator $B^{(\mathrm{H})} \circ J$, and with $C:=\left\|B^{(\mathrm{H})}\right\|_{L^{2}(0,1) \rightarrow \ell^{2}}=\sqrt{\pi}$ (cf. [13]) we have the upper estimate $\sigma_{i}\left(B^{(\mathrm{H})} \circ\right.$ $J) \leq \sqrt{\pi} \sigma_{i}(J)(i=1,2, \ldots)$.

To the best of our knowledge, to date no examples are known that show a violation of $\sigma_{i}(B \circ D) \asymp \sigma_{i}(D)$. In the present study we shall show that $\sigma_{i}\left(B^{(\mathrm{H})} \circ J\right) / \sigma_{i}(J) \leq C i^{-1 / 2}$

\footnotetext{
${ }^{1}$ We shall measure the decay rates of the singular values asymptotically; thus for decreasing sequences $s_{i} \geq 0$ and $t_{i} \geq 0$, we say that $s_{i} \asymp t_{i}$ as $i \rightarrow \infty$ if there are constants $0<\underline{c} \leq \bar{c}<\infty$ such that the inequalities

$$
\underline{c} s_{i} \leq t_{j} \leq \bar{c} s_{i} \quad(i=1,2, \ldots)
$$
}

are valid. 


\section{ETNA}

Kent State University and

Johann Radon Institute (RICAM)

$(i=1,2, \ldots)$ with some positive constant $C$, and the non-compact Hausdorff moment operator $B^{(\mathrm{H})}$ enlarges the degree of ill-posedness of $J$ by a factor $1 / 2$, at least.

We shall start in Section 2 with some results for general operators, relating conditional stability estimates to the decay of the singular numbers of the composition $B \circ D$. Conditional stability estimates for the composition with the Hausdorff moment operator are given in Section 3, for both the embedding operator and the integration operator. According to Theorem 2.1 we derive lower bounds for the decay rates of the compositions $B^{(\mathrm{H})} \circ \mathcal{E}^{(k)}$ and $B^{(\mathrm{H})} \circ J$, respectively.

The composite operators, both $A^{*} A$ for $A=B^{(\mathrm{H})} \circ J$ and $\widetilde{A}^{*} \widetilde{A}$ for $\widetilde{A}=B^{(\mathrm{M})} \circ J$, are Hilbert-Schmidt operators, because the factor $J$ is such. In particular, these may be expressed as linear Fredholm integral operators acting in $L^{2}(0,1)$ with symmetric positive kernels $k$ and $\widetilde{k}$, respectively. There are well-known results which state that a certain type of kernel smoothness yields a minimum decay rate of the corresponding singular values of the integral operator. Therefore, in Section 4 we establish the form of the kernels $k$ and $\widetilde{k}$, and we study their smoothness. In particular, for the composition $B^{(\mathrm{H})} \circ J$ we shall see that the known results are not applicable, whereas in the case $B^{(\mathrm{M})} \circ J$ these known results are in alignment with $\sigma_{i}\left(B^{(\mathrm{M})} \circ J\right) \asymp \sigma_{i}(J) \asymp 1 / i$.

Finally, in Section 5, we improve the upper bounds for the decay of the singular values of the composition $B^{(\mathrm{H})} \circ J$, giving the first example that violates $\sigma_{i}(B \circ D) \asymp \sigma_{i}(D)$ as $i \rightarrow \infty$ in the context of a non-compact outer operator $B$. This approach bounds the singular values by means of bounds for the Hilbert-Schmidt norm of the composition $\left\|\left(B^{(\mathrm{H})} \circ J\right)\left(I-Q_{n}\right)\right\|_{\mathrm{HS}}$, where $Q_{n}$ is a projection on the $n$-dimensional subspace of adapted Legendre polynomials in $L^{2}(0,1)$. We continue to discuss the result obtained in Section 6. An appendix completes the paper.

2. Results for general operators. We start with a general theorem explaining the interplay of conditional stability estimates and upper bounds for the degree of ill-posedness. To this end we shall use results from the theory of $s$-numbers, and we refer to the monograph [18, Proposition 2.11.6]. In particular, for a compact operator, say $T: X \rightarrow Y$, the singular values $\sigma_{i}(T)$ coincide with the corresponding (linear) approximation numbers $a_{i}(T)$, and hence the identities

$$
\sigma_{i}(T)=\left\|T\left(I-P_{i-1}\right)\right\|_{X \rightarrow Y}=\inf \left\{\|T-L\|_{X \rightarrow Y}: \operatorname{dim}(\mathcal{R}(L))<i\right\}
$$

hold for all $i=1,2, \ldots$ Above, we denote by $\left\{\sigma_{i}(T), u_{i}, v_{i}\right\}_{i=1}^{\infty}$, with $T u_{i}=\sigma_{i}(T) v_{i}$ $(i=1,2, \ldots)$, the well-defined (monotonic) singular system of the compact operator $T$, and by $P_{n}: X \rightarrow X(n=1,2, \ldots)$ the orthogonal projection onto $\operatorname{span}\left(u_{1}, \ldots, u_{n}\right)$, the $n$-dimensional subspace of $X$, where we assign $P_{0}=0: X \rightarrow X$.

The main estimate is as follows:

THEOREM 2.1. Let $D: X \rightarrow Z$ and $A: X \rightarrow Y$ be compact linear operators between the infinite-dimensional Hilbert spaces $X, Y$, and $Z$ with non-closed ranges $\mathcal{R}(D)$ and $\mathcal{R}(A)$. Suppose that there exists an index function $\Psi:(0, \infty) \rightarrow(0, \infty)$ such that, for $0<\delta \leq\|A\|_{X \rightarrow Y}$, the conditional stability estimate

$$
\sup \left\{\|D x\|_{Z}:\|A x\|_{Y} \leq \delta,\|x\|_{X} \leq 1\right\} \leq \Psi(\delta)
$$

holds. Then we have

$$
\sigma_{i}(D) \leq \Psi\left(\sigma_{i}(A)\right) \quad(i=1,2, \ldots)
$$

and also

$$
\Psi^{-1}\left(\sigma_{i}(D)\right) \leq \sigma_{i}(A) \quad(i=1,2, \ldots)
$$


If the operators $D^{*} D: X \rightarrow X$ and $A^{*} A: X \rightarrow X$ commute, and if the index function $t \mapsto \Psi^{2}(\sqrt{t}), t>0$, is concave, then the converse holds true in the sense that (2.3) implies the stability estimate (2.2).

Proof. Suppose that (2.2) holds true. Then for every $u \in X,\|u\|_{X} \leq 1$, we see that

$$
\|A u\|_{Y} \leq \delta \quad \text { implies that } \quad\|D u\|_{Z} \leq \Psi(\delta) \quad(\delta>0) .
$$

Consider the singular projections $P_{i}$ for the operator $A$. For arbitrarily chosen $x \in X$ with $\|x\|_{X} \leq 1$, we see that

$$
\left\|A\left(I-P_{i-1}\right) x\right\|_{Y} \leq\left\|A\left(I-P_{i-1}\right)\right\|_{X \rightarrow Y}\|x\|_{X} \leq \sigma_{i}(A) .
$$

Applying (2.5) with $u:=\left(I-P_{i-1}\right) x$ and $\delta:=\sigma_{i}(A)$ yields $\left\|D\left(I-P_{i-1}\right) x\right\|_{Z} \leq \Psi\left(\sigma_{i}(A)\right)$. Since $x \in X$ with $\|x\|_{X} \leq 1$ was chosen arbitrarily, we even arrive at $\left\|D\left(I-P_{i-1}\right)\right\|_{X \rightarrow Y} \leq$ $\Psi\left(\sigma_{i}(A)\right)$.

By virtue of (2.1) we find for

$$
\sigma_{i}(D)=\inf \left\{\|D-L\|_{X \rightarrow Z}: \operatorname{dim}(\mathcal{R}(L))<i\right\}
$$

that

$$
\sigma_{i}(D) \leq\left\|D\left(I-P_{i-1}\right)\right\|_{X \rightarrow Z} \leq \Psi\left(\sigma_{i}(A)\right),
$$

which proves (2.3). Since the inverse of an index function exists and is also an index function, hence monotonically increasing, the estimate (2.4) is a consequence of (2.3).

Next, suppose that the operators $D^{*} D$ and $A^{*} A$ commute, and hence they share the same singular functions $u_{1}, u_{2}, \ldots$. Clearly, for $x=0$ we have that $\|D x\|_{Z}=0 \leq \Psi(\delta)$, so we may and do assume that $x \neq 0$. Assume that (2.3) holds. We abbreviate $f(t):=\Psi^{2}(\sqrt{t})$, $t>0$. First, if $\|x\|_{X}=1$ then we bound

$$
\begin{aligned}
\|D x\|_{Z}^{2} & =\sum_{i=1}^{\infty} \sigma_{i}^{2}(D)\left|\left\langle x, u_{i}\right\rangle\right|^{2} \leq \sum_{i=1}^{\infty} f\left(\sigma_{i}^{2}(A)\right)\left|\left\langle x, u_{i}\right\rangle\right|^{2} \\
& \leq f\left(\sum_{i=1}^{\infty} \sigma_{i}^{2}(A)\left|\left\langle x, u_{i}\right\rangle\right|^{2}\right)=f\left(\|A x\|_{Y}^{2}\right),
\end{aligned}
$$

where we used Jensen's Inequality for $f$. Hence $\|D x\|_{Z} \leq \Psi\left(\|A x\|_{Y}\right)$. Consequently, for $x \in X,\|x\|_{X}>0$, this extends to

$$
\frac{\|D x\|_{Z}}{\|x\|_{X}} \leq \Psi\left(\frac{\|A x\|_{Y}}{\|x\|_{X}}\right), \quad x \neq 0 .
$$

For the concave index function $f$ we see that $f(a t) \geq a f(t), t>0$, whenever $a \leq 1$. Thus for $a:=\|x\|_{X}^{2} \leq 1$ and $t:=\left(\|A x\|_{Y} /\|x\|_{X}\right)^{2}$ we find that

$$
\|D x\|_{Z} \leq \Psi\left(\|A x\|_{Y}\right), \quad x \neq 0,
$$

which in turn yields the validity of (2.2), and this completes the proof.

REMARK 2.2. If the conditional stability estimate (2.2) is not valid for all $\delta>0$, but is valid for sufficiently small $\delta>0$, then the estimates (2.3) and (2.4) are not valid for all $i \in \mathbb{N}$, but are valid for $i$ sufficiently large. Hence, the corresponding assertions about the singular value asymptotics do not change.

REMARK 2.3. We mention here that the term

$$
\sup \left\{\|D x\|_{Z}:\|A x\|_{Y} \leq \delta,\|x\|_{X} \leq 1\right\},
$$


occurring in formula (2.2), is a special case of the modulus of continuity

$$
\omega_{M}(\delta):=\sup \left\{\|D x\|_{Z}:\|A x\|_{Y} \leq \delta, x \in M\right\},
$$

with some closed and bounded set $M \subset X$ such that $D M$ represents a compact set of $Z$. This is due to the compactness of the operator $D: X \rightarrow Z$. Note that $\omega_{M}(\delta)$ is increasing in $\delta>0$ with the limit condition $\lim _{\delta \rightarrow 0} \omega_{M}(\delta)=0$. Moreover, we have for constants $E>1$ and centrally symmetric and convex sets $M$ that $\omega_{E M}(\delta)=E \omega_{M}(\delta / E)$. For further details of this concept, we refer, for example, to [4, 10]. In general, one is interested in bounding the modulus of continuity by a majorant index function $\Psi$ as in formula (2.2), which leads to conditional stability estimates. Precisely, in (2.2) we have the situation of a centrally symmetric and convex $M=\left\{x \in X:\|x\|_{X} \leq 1\right\}$ under consideration with associated majorant index function $\Psi$. Consequently, this also yields, for $E>1$,

$$
\sup \left\{\|D x\|_{Z}:\|A x\|_{Y} \leq \delta,\|x\|_{X} \leq E\right\} \leq E \Psi(\delta / E)
$$

It is known from approximation theory, and it was highlighted in [4, Proposition 2.9], that there is always a concave majorant for the modulus of continuity, such that without loss of generality we may assume $\Psi$ to be concave.

REMARK 2.4. It is shown in [4, Theorem 4.1] that the required concavity of the function $t \mapsto \Psi^{2}(\sqrt{t}), t>0$, automatically holds true whenever $D^{*} D$ is a function of $A^{*} A$, i.e., $D^{*} D=\varphi\left(A^{*} A\right)$ for an index function $\varphi$. For power-type functions $\Psi$, the concavity assertion holds true if and only if $\Psi$ is concave; see the end of Remark 2.3.

3. Compositions with the Hausdorff moment operator. In order to apply Theorem 2.1 to compositions with the integration operator $B^{(\mathrm{H})}$ from $(1.7)$, we formulate appropriate conditional stability estimates.

THEOREM 3.1. There are constants $C_{k}>0$ depending on $k=0,1,2, \ldots$ such that:

(a) For the composite problem $B^{(\mathrm{H})} \circ \mathcal{E}^{(k)}$, the bound

$$
\sup \left\{\|x\|_{L^{2}(0,1)}:\left\|B^{(\mathrm{H})}\left(\mathcal{E}^{(k)} x\right)\right\|_{\ell^{2}} \leq \delta,\|x\|_{H^{k}(0,1)} \leq 1\right\} \leq \frac{C_{k}}{(\ln (1 / \delta))^{k}}
$$

holds for sufficiently small $\delta>0$.

(b) For the composite problem $B^{(\mathrm{H})} \circ J$, the bound

$$
\sup \left\{\|J x\|_{L^{2}(0,1)}:\left\|B^{(H)}(J x)\right\|_{\ell^{2}} \leq \delta,\|x\|_{L^{2}(0,1)} \leq 1\right\} \leq \frac{C_{0}}{\ln (1 / \delta)}
$$

holds for sufficiently small $\delta>0$.

The proof will be along the lines of [22], and we shall state the key points here. The analysis will be based on the (normalized) shifted Legendre polynomials, $\left\{L_{j}\right\}_{j=1}^{\infty}$, with the explicit representation

$$
L_{j}(t)=\frac{\sqrt{2 j-1}}{(j-1) !}\left(\frac{\mathrm{d}}{\mathrm{d} t}\right)^{j-1} t^{j-1}(1-t)^{j-1} \quad(t \in[0,1], j=1,2, \ldots) .
$$

The system $\left\{L_{j}\right\}_{j=1}^{\infty}$ is the result of the Gram-Schmidt orthonormalization process of the system $\left\{t^{j-1}\right\}_{j=1}^{\infty}$ of monomials. Consequently, we have

$$
\operatorname{span}\left(1, t, \ldots, t^{N-1}\right)=\operatorname{span}\left(L_{1}, L_{2}, \ldots, L_{N}\right)
$$


These polynomials form an orthonormal basis in $L^{2}(0,1)$, and we denote by $Q_{n}$ the orthogonal projections onto the span $\mathcal{D}\left(Q_{n}\right) \subset L^{2}(0,1)$ of the first $n$ Legendre polynomials, and by $P_{n}$ the projection onto the first $n$ unit basis vectors in $\ell^{2}$.

LEMMA 3.2. For the Hausdorff moment operator $B=B^{(\mathrm{H})}$ from (1.7), the following hold true.

(I) $P_{n} B Q_{n}=P_{n} B$,

(II) $P_{n} B B^{*} P_{n}=H_{n}$ with $H_{n}: \ell_{n}^{2} \rightarrow \ell_{n}^{2}$ being the $n$-dimensional segment of the Hilbert matrix,

(III) $\left\|Q_{n} x\right\|_{L^{2}(0,1)} \leq\left\|P_{n} B x\right\|_{\ell^{2}} / \sigma_{n}\left(P_{n} B\right)$, and

(IV) $\sigma_{n}\left(P_{n} B\right)=\left\|H_{n}^{-1}\right\|_{\ell_{n}^{2} \rightarrow \ell_{n}^{2}}^{1 / 2}$.

Consequently we have that $\left\|Q_{n} x\right\|_{L^{2}(0,1)} \leq\left\|H_{n}^{-1}\right\|_{\ell_{n}^{2} \rightarrow \ell_{n}^{2}}^{1 / 2}\left\|P_{n} B x\right\|_{\ell^{2}}$.

Proof. The first assertion (I) is easily checked and it results from the fact that the GramSchmidt matrix for turning from the monomials to the Legendre coefficients (see (3.4)) is lower triangular. The second assertion (II) was shown in [7, Proposition 4]. The final assertion (IV) is a restatement of

$$
\sigma_{n}\left(P_{n} B\right) \leq \frac{\left\|P_{n} B x\right\|_{\ell^{2}}}{\left\|Q_{n} x\right\|_{L^{2}(0,1)}}, \quad x \neq 0 .
$$

In view of the first item (I), it is enough to prove that

$$
\sigma_{n}\left(P_{n} B\right) \leq \inf _{0 \neq z \in \mathcal{D}\left(Q_{n}\right)} \frac{\left\|P_{n} B z\right\|_{\ell^{2}}}{\|z\|_{L^{2}(0,1)}} .
$$

It is well known from approximation theory that

$$
\sigma_{n}\left(P_{n} B\right)=\inf _{X_{n}: \operatorname{dim}\left(X_{n}=n\right)} \inf _{0 \neq z \in \mathcal{D}\left(Q_{n}\right)} \frac{\left\|P_{n} B z\right\|_{\ell^{2}}}{\|z\|_{L^{2}(0,1)}} .
$$

Indeed, the right-hand side above corresponds to the definition of the Bernstein numbers, which constitute an $s$-number (see [17, Theorem 4.5]), and this proves item (III). The last item (IV) follows from

$$
\sigma_{n}^{2}\left(P_{n} B\right)=\sigma_{n}\left(P_{n} B B^{*} P_{n}\right)=\sigma_{n}\left(P_{n} H P_{n}\right)=\sigma_{n}\left(H_{n}\right)=\frac{1}{\left\|H_{n}^{-1}\right\|_{\ell_{n}^{2} \rightarrow \ell_{n}^{2}}},
$$

which in turn yields the final assertion. The proof is complete.

The next result concerns the approximation power of smooth functions by Legendre polynomials.

LEMMA 3.3. For functions $x \in H^{k}(0,1)$, there is a constant $K_{k}$ such that

$$
\left\|\left(I-Q_{n}\right) x\right\|_{L^{2}(0,1)} \leq K_{k} \frac{1}{n^{k}} \quad(n \in \mathbb{N}) .
$$

For $k=1$, and hence $x \in H^{1}(0,1)$, this my be specified as

$$
\left\|\left(I-Q_{n}\right) x\right\|_{L^{2}(0,1)} \leq \frac{\left\|x^{\prime}\right\|_{L^{2}(0,1)}}{2 n} \quad(n \in \mathbb{N}) .
$$

REMARK 3.4. In [2, Theorem 4.1], the proof of (3.5) is given for $k=1$. In [2, Remark 4.1], the extension for other values of $k$ is stated without explicit proof. In [25, 
Theorem 2.5] a proof is given for the Legendre polynomials on the interval $(-1,1)$, based on [25, Theorem 2.1], which describes the decay rates of the expansions in terms of Legendre polynomials for functions with Sobolev-type smoothness. The specification in the second bound is taken from [22, equation (27)].

Based on the above preparations we turn to the proof.

Proof of Theorem 3.1. For both assertions (a) and (b) we are going to use a decomposition of the form

$$
\|z\|_{L^{2}(0,1)} \leq\left\|Q_{n} z\right\|_{L^{2}(0,1)}+\left\|\left(I-Q_{n}\right) z\right\|_{L^{2}(0,1)},
$$

where $Q_{n}$ is the orthogonal projection on the span of the first $n$ Legendre polynomials.

For the first assertion (a), we let $z:=x$, and we bound each summand. Recall that here $\mathcal{E}^{(k)}$ is the natural embedding, with $\mathcal{E}^{(k)} x=x$ for all $x \in H^{k}(0,1)$. Thus, by Lemma 3.2 the first summand is bounded as

$$
\left\|Q_{n} x\right\|_{L^{2}(0,1)} \leq \delta\left\|H_{n}^{-1}\right\|_{\ell_{n}^{2} \rightarrow \ell_{n}^{2}}^{1 / 2} .
$$

From $[3,23,26]$ we know that there is a constant $\widehat{C}$, independent of $n$, for which

$$
\left\|H_{n}^{-1}\right\|_{\ell_{n}^{2} \rightarrow \ell_{n}^{2}} \leq \widehat{C} \exp (4 \ln (1+\sqrt{2}) n) \leq \widehat{C} \exp (4 n) .
$$

This yields

$$
\left\|Q_{n} x\right\|_{L^{2}(0,1)} \leq \sqrt{\widehat{C}} \exp (2 n) \delta .
$$

The second summand in (3.6) is bounded in Lemma 3.3, and altogether we find that

$$
\|x\|_{L^{2}(0,1)} \leq \sqrt{\widehat{C}} \exp (2 n) \delta+K_{k} \frac{1}{n^{k}} .
$$

We choose an integer $N=n(\delta)$ such that the two terms on the right-hand side of the estimate (3.8) are equilibrated. This is achieved by letting $N$ be given from

$$
N=\left\lfloor\frac{1}{4} \ln (1 / \delta)\right\rfloor+1, \quad 0<\delta \leq \exp (-4) .
$$

Substituting $n:=N$ in (3.8) yields, for sufficiently small $\delta>0$, the final estimate

$$
\|x\|_{L^{2}(0,1)} \leq \frac{C_{k}}{(\ln (1 / \delta))^{k}},
$$

with some positive constant $C_{k}$ depending on $k$.

To prove the second assertion (b), we assign $z:=J x$. Then the first summand in (3.6) allows for an estimate of the form

$$
\left\|Q_{n}(J x)\right\|_{L^{2}(0,1)} \leq \sqrt{\widehat{C}} \exp (2 n) \delta,
$$

again for some constant $\widehat{C}>0$. For bounding $\left\|\left(I-Q_{n}\right)(J x)\right\|_{L^{2}(0,1)}$, we use the second estimate in Lemma 3.3, which gives, for $\|x\|_{L^{2}(0,1)} \leq 1$, the bound

$$
\left\|\left(I-Q_{n}\right)(J x)\right\|_{L^{2}(0,1)} \leq \frac{\|x\|_{L^{2}(0,1)}}{2 n} \leq \frac{1}{2 n} .
$$

Then we can proceed as for the first assertion in order to complete the proof of the second assertion, and of the theorem. 
The proof formulated above is an alternative to the proof of [7, Theorem 1] for $k=1$ and an extension to the cases $k=2,3, \ldots$ Consequences of Theorems 2.1 and 3.1 for the singular value decay rate of the Hausdorff moment composite operator $A:=B^{(\mathrm{H})} \circ \mathcal{E}^{(k)}$ are summarized in the following corollary.

COROLLARY 3.5. For the composite Hausdorff moment problem $B^{(\mathrm{H})} \circ \mathcal{E}^{(k)}$ there exist positive constants $C_{k}, \underline{C}$, and $\bar{C}$ such that

$$
\exp (-\underline{C} i) \leq \exp \left(-\left(\frac{C_{k}}{\sigma_{i}\left(\mathcal{E}^{(k)}\right)}\right)^{1 / k}\right) \leq \sigma_{i}\left(B^{(\mathrm{H})} \circ \mathcal{E}^{(k)}\right) \leq \sqrt{\pi} \sigma_{i}\left(\mathcal{E}^{(k)}\right) \leq \frac{\bar{C}}{i^{k}}
$$

is valid for sufficiently large indices $i \in \mathbb{N}$.

Proof. Taking into account the well-known singular value asymptotics $\sigma_{i}\left(\mathcal{E}^{(k)}\right) \asymp i^{-k}$ as $i \rightarrow \infty$ (cf. [14, Section 3.c]) and the norm $\left\|B^{(\mathrm{H})}\right\|_{L^{2}(0,1) \rightarrow \ell^{2}}=\sqrt{\pi}$, we simply find for the composition $A=B^{(\mathrm{H})} \circ \mathcal{E}^{(k)}$ the estimates from above,

$$
\sigma_{i}\left(B^{(\mathrm{H})} \circ \mathcal{E}^{(k)}\right) \leq \sqrt{\pi} \sigma_{i}\left(\mathcal{E}^{(k)}\right) \leq \frac{\bar{C}}{i^{k}}
$$

with some positive constant $\bar{C}$.

We need to show the lower bounds, and we are going to apply Theorem 2.1 in combination with the estimate (3.1) from Theorem 3.1. To do so we set $X:=H^{k}(0,1), Z:=L^{2}(0,1)$, and $Y:=\ell^{2}$, as well as $D:=\mathcal{E}^{(k)}, A:=B^{(\mathrm{H})} \circ \mathcal{E}^{(k)}$, and $\Psi(\delta):=C_{k} /(\ln (1 / \delta))^{k}$ for sufficiently small $\delta>0$. This last function has the inverse $\Psi^{-1}(t)=\exp \left(-\left(C_{k} / t\right)^{1 / k}\right)$. Then the conditional stability estimate (2.2) attains the form (3.1), and we derive from (2.4) that

$$
\exp \left(-\left(\frac{C_{k}}{\sigma_{i}(D)}\right)^{1 / k}\right)=\Psi^{-1}\left(\sigma_{i}\left(\mathcal{E}^{(k)}\right)\right) \leq \sigma_{i}\left(B^{(\mathrm{H})} \circ \mathcal{E}^{(k)}\right)
$$

for sufficiently large indices $i \in \mathbb{N}$. This completes the proof.

Theorem 2.1 also applies to the composition $B^{(\mathrm{H})} \circ J$, and, along the lines of the proof of Corollary 3.5, yields the following result.

COROLLARY 3.6. For the composite Hausdorff moment problem $B^{(\mathrm{H})} \circ J$ there exist positive constants $\underline{C}$ and $\bar{C}$ such that

$$
\exp (-\underline{C} i) \leq \sigma_{i}\left(B^{(\mathrm{H})} \circ J\right) \leq \frac{\bar{C}}{i}
$$

is valid for sufficiently large indices $i \in \mathbb{N}$.

REMARK 3.7. The composite mapping $B^{(\mathrm{H})} \circ J$ may be viewed as a forward mapping when reconstructing the derivative of an unknown function from Hausdorff moments, captured in $B^{(\mathrm{H})}$. The authors in $[15,27]$ have discussed the reconstruction of an unknown function from Legendre moments, which will correspond to a composite mapping $B^{(\mathrm{L})} \circ J$, with $B^{(\mathrm{L})}: L^{2}(0,1) \rightarrow \ell^{2}$, assigning to a function $g \in L^{2}(0,1)$ the sequence of moments $\int_{0}^{1} g(t) L_{i}(t) \mathrm{d} t(i=1,2, \ldots)$. In this case the mapping $B^{(\mathrm{L})}$ constitutes a (noncompact) unitary operator, and we will have $\sigma_{i}\left(B^{(\mathrm{L})} \circ J\right)=\sigma_{i}(J) \asymp 1 / i(i=1,2, \ldots)$.

The gap between the lower and upper bounds for the singular values $\sigma_{i}\left(B^{(\mathrm{H})} \circ \mathcal{E}^{(k)}\right)$ and $\sigma_{i}\left(B^{(\mathrm{H})} \circ J\right)$ expressed in Corollaries 3.5 and 3.6, respectively, is quite large. This gap does not allow us to decide whether the composite problems are moderately ill-posed (when the upper bounds are realistic), or severely (exponentially) ill-posed (when the lower bounds are realistic) (cf., e.g., [9, Definition 8]). 


\section{ETNA}

Kent State University and

Johann Radon Institute (RICAM)

4. Discussion of kernel smoothness. The composite operators that were considered so far are Hilbert-Schmidt operators, because the factors $J$ and $\mathcal{E}^{(k)}$, respectively, have this property. Hilbert-Schmidt operators acting in $L^{2}(0,1)$ are integral operators, and hence these can be given in the form of a Fredholm integral operator $[G(x)](s):=\int_{0}^{1} k(s, t) x(t) \mathrm{d} t$ $(0 \leq s \leq 1)$ with kernel $k=k(s, t) \in L^{2}((0,1) \times(0,1))$.

It is well known that decay rates of the singular values grow with the smoothness of the kernel $k$, and we refer in this context to the following result.

Lemma 4.1 (see [5]). Consider in $L^{2}(0,1)$ the Fredholm integral operator $[G(x)](s):=$ $\int_{0}^{1} k(s, t) x(t) \mathrm{d} t(0 \leq s \leq 1)$ and assume that the kernel $k$ and the derivatives $\partial k / \partial s, \ldots$, $\partial^{l-1} k / \partial s^{l-1}$ exist and are continuous in $s$ for almost all $t$. Moreover, assume that there exist $g \in L^{2}((0,1) \times(0,1))$ and $V \in L^{1}(0,1)$ such that

$$
\frac{\partial^{l} k(s, t)}{\partial s^{l}}=\int_{0}^{s} g(\tau, t) \mathrm{d} \tau+V(t)
$$

Then we have

$$
\sigma_{i}(G)=o\left(i^{-l-1.5}\right) \quad \text { as } i \rightarrow \infty .
$$

We emphasize that Lemma 4.1 provides us with upper rate bounds, corresponding to a minimum speed of the decay to zero of the singular values. If, in particular, the kernel is infinitely smooth on the whole unit square, then the decay rate of the associated singular values is faster than $\mathcal{O}\left(i^{-\eta}\right)$ for arbitrarily large $\eta>0$. Consequently, an exponential-type decay of the singular values can take place. Lower bounds cannot be expected in general, as shown by the simple rank-one example $k(s, t)=(s-1 / 2)_{+} \times(t-1 / 2)_{+}(0 \leq s, t \leq 1)$, which exhibits low smoothness, but the sequence of singular values with $\sigma_{1}=1$ and $\sigma_{i}=0$ $(i=2,3, \ldots)$ decays at any rate. However, non-smoothness aspects like non-differentiability, non-Lipschitz, and occurring poles in the kernel give limitations for the decay rate of the singular values. So we are not aware of examples of exponentially ill-posed linear problems with kernel $k$ that does not belong to $C^{\infty}([0,1] \times[0,1])$.

Below, we shall determine the kernels $k$ and $\tilde{k}$ of the self-adjoint companions $A^{*} A$ and $\widetilde{A}^{*} \widetilde{A}$ of the compositions $A:=B^{(\mathrm{H})} \circ J: L^{2}(0,1) \rightarrow \ell^{2}$ (with the Hausdorff moment operator) and $\widetilde{A}:=B^{(\mathrm{M})} \circ J: L^{2}(0,1) \rightarrow L^{2}(0,1)$ (with a multiplication operator), respectively.

For the first composition, we have the following proposition, the proof of which is given in the appendix.

PROPOSITION 4.2. The kernel $k$ of the Fredholm integral operator $A^{*} A$ mapping in $L^{2}(0,1)$ with $A=B^{(\mathrm{H})} \circ J$ attains the form

$$
k(s, t)=\sum_{j=1}^{\infty} \frac{\left(1-s^{j}\right)\left(1-t^{j}\right)}{j^{2}} \quad(0 \leq s, t \leq 1) .
$$

The second composition $\widetilde{A}:=B^{(\mathrm{M})} \circ J$ with multiplier function $m(t)=t^{\theta}$ for $\theta>0$ constitutes a linear Volterra integral operator. However, it can be rewritten as a linear Fredholm integral operator,

$$
[\widetilde{A} x](s)=\int_{0}^{1} \kappa(s, t) x(t) \mathrm{d} t, \quad \text { with } \kappa(s, t)= \begin{cases}s^{\theta} & (0 \leq t \leq s \leq 1), \\ 0 & (0 \leq s<t \leq 1)\end{cases}
$$

and we refer to $[6,11]$ for further investigations. Taking into account that $\kappa(t, s)$ with switched variables is the kernel of the adjoint integral operator $\widetilde{A}^{*}$, we have that the kernel $k$ of the 
operator $\widetilde{A}^{*} \widetilde{A}$ mapping in $L^{2}(0,1)$ is given as

$$
\tilde{k}(s, t)=\int_{0}^{1} \kappa(\tau, s) \kappa(\tau, t) \mathrm{d} \tau .
$$

This yields the following proposition for the second composition case.

PROPOSITION 4.3. The kernel $\tilde{k}$ of the Fredholm integral operator $\widetilde{A}^{*} \widetilde{A}$ mapping in $L^{2}(0,1)$ with $\widetilde{A}$ from (4.4) attains the form

$$
\tilde{k}(s, t)=\int_{\max (s, t)}^{1} \tau^{2 \theta} \mathrm{d} \tau=1-\frac{\max (s, t)^{2 \theta+1}}{2 \theta+1} \quad(0 \leq s, t \leq 1) .
$$

We are going to discuss the implications of Lemma 4.1 on the decay rates of the singular values of both $A^{*} A$ and $\widetilde{A}^{*} \widetilde{A}$. We start with the latter.

The kernel $\tilde{k}$ from (4.5) is continuous and satisfies, for all $\theta>0$, the Lipschitz condition $\tilde{k} \in \operatorname{Lip}_{1}([0,1] \times[0,1])$, which means that there is a constant $L>0$ such that, for all $s, \hat{s}, t, \hat{t} \in[0,1]$,

$$
|\tilde{k}(s, t)-\tilde{k}(\hat{s}, \hat{t})| \leq L(|s-\hat{s}|+|t-\hat{t}|) .
$$

The author in [21] proves that in this case we can guarantee the decay rate

$$
\sigma_{i}\left(\widetilde{A}^{*} \widetilde{A}\right)=\mathcal{O}\left(i^{-2}\right) \quad \text { as } i \rightarrow \infty .
$$

The kernel $\tilde{k}$ from (4.5), containing a maximum term, is not differentiable at the diagonal of the unit square. If it were continuously differentiable on $[0,1] \times[0,1]$, then the decay rate would even be improved to $\sigma_{i}\left(\widetilde{A}^{*} \widetilde{A}\right)=o\left(i^{-2}\right)$, and we refer to [20]. Indeed, the exact asymptotics $\sigma_{i}\left(\widetilde{A}^{*} \widetilde{A}\right) \asymp i^{-2}$ for all $\theta>0$ was shown in [11] .

We turn to discussing the singular values of the operator $A^{*} A$ with kernel $k$ from (4.3). Since the series $\sum_{j=1}^{\infty}\left(1-s^{j}\right)\left(1-t^{j}\right) / j^{2}$ of continuous functions is uniformly absolutely convergent, the kernel $k$ actually belongs to the space $C([0,1] \times[0,1])$ of continuous functions. This allows for partial differentiation with respect to $s$ as

$$
k_{s}(s, t)=\sum_{j=1}^{\infty} \frac{-s^{j-1}\left(1-t^{j}\right)}{j} .
$$

Figure 4.1 presents a plot of the kernel $k$ and its first partial derivative $k_{s}$.
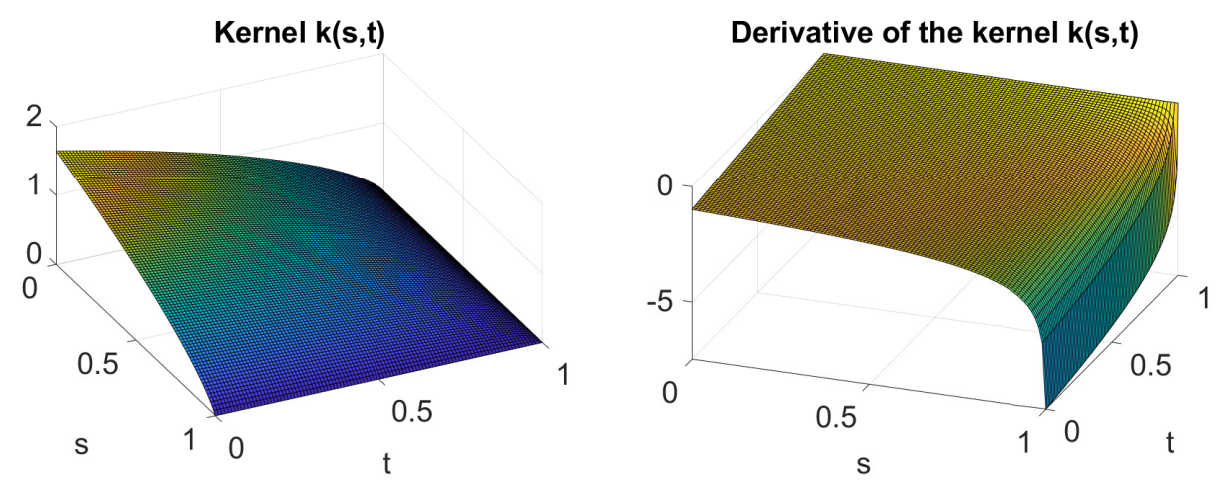

FIG. 4.1. Plot of the kernel $k$ and its derivative $k_{s}$. 
The right picture shows that the partial derivative has a pole at the right boundary with $s=1$ of the unit square. This pole implies that $k \notin \operatorname{Lip}_{1}([0,1] \times[0,1])$. On the other hand, $k_{s}$ is smooth elsewhere and allows for further partial differentiation with a second partial derivative

$$
k_{s s}(s, t)=\sum_{j=2}^{\infty} \frac{-(j-1) s^{j-2}\left(1-t^{j}\right)}{j},
$$

which also has a pole at $s=1$. We note that the order of the pole there grows by one for every higher partial differentiation step with respect to $s$.

Based on (4.7) one can derive, in light of formula (4.1) from Lemma 4.1, that

$$
\frac{\partial k(s, t)}{\partial s}=\int_{0}^{s} g(\tau, t) \mathrm{d} \tau+V(t)
$$

with $g(\tau, t)=\sum_{j=2}^{\infty}-(j-1) s^{j-2}\left(1-t^{j}\right) / j$ and $V(t)=t-1$. Notice that $g \notin L^{2}((0,1) \times$ $(0,1))$, which prevents the application of Lemma 4.1, even in the case $l=1$. Thus, Lemma 4.1 is not applicable, and we may not make inference on the decay rates of the singular values by means of considering kernel smoothness.

REMARK 4.4. We have not found assertions in the literature that handle the situation of such poles in light of decay rates of singular values.

In summary, the smoothness of the kernel $k$ from (4.3) is strongly limited. In particular we have $k \notin C^{\infty}([0,1] \times[0,1])$. This makes an exponential decay rate of the singular values $\sigma_{i}(A)$ appear rather unlikely. However, at present we have no analytical approach to check this in more detail.

5. Bounding the singular values of the composite operator $\boldsymbol{B}^{(\mathrm{H})} \circ J$. Our aim of this section is to improve the upper bound in (3.9) for the singular values $\sigma_{i}(A)=\sigma_{i}\left(B^{(\mathrm{H})} \circ J\right)$ of the composite operator

$$
\text { A: } L^{2}(0,1) \stackrel{J}{\longrightarrow} L^{2}(0,1) \stackrel{B^{(\mathrm{H})}}{\longrightarrow} \ell^{2} .
$$

We emphasize that this composition constitutes a Hilbert-Schmidt operator, since its component $J$ is Hilbert-Schmidt, and our argument will be based on bounding the Hilbert-Schmidt norm

$$
\|A\|_{\mathrm{HS}}=\left(\sum_{i=1}^{\infty} \sigma_{i}^{2}(A)\right)^{1 / 2} .
$$

The main result will be the following.

THEOREM 5.1. For the composite Hausdorff moment problem $B^{(\mathrm{H})} \circ J$ with operators $J$ from (1.4) and $B^{(\mathrm{H})}$ from (1.7), there exists a positive constant $C$ such that

$$
\sigma_{i}\left(B^{(\mathrm{H})} \circ J\right) \leq \frac{C}{i^{3 / 2}} \quad(i \in \mathbb{N}) .
$$

Consequently, there is some constant $K>0$ such that

$$
\sigma_{i}\left(B^{(\mathrm{H})} \circ J\right) / \sigma_{i}(J) \leq \frac{K}{i^{1 / 2}} \quad(i \in \mathbb{N}) .
$$

For its proof we make the following preliminary considerations. We recall the definition of the shifted Legendre polynomials $L_{j}(j=1,2, \ldots)$ from (3.3), as well as $Q_{n}: L^{2}(0,1) \rightarrow$ 
$L^{2}(0,1)$, being the orthogonal projection onto the $n$-dimensional subspace of the polynomials up to degree $n-1$.

For the further estimates, the next result is important. Here, we denote by

$$
\left.\left\{\sigma_{i}(A), u_{i}, v_{i}\right)\right\}_{i=1}^{\infty}
$$

the singular system of the compact operator $A=B^{(\mathrm{H})} \circ J$.

PROPOSITION 5.2. Let $Q_{n}$ denote the projections onto $\operatorname{span}\left\{L_{1}, \ldots, L_{n}\right\}$ of the Legendre polynomials up to degree $n-1$, and let $P_{n}$ be the singular projection onto $\operatorname{span}\left\{u_{1}, \ldots, u_{n}\right\}$ of the first $n$ eigenelements of $A$. Then we have for $A=B^{(\mathrm{H})} \circ J$ that

$$
\sum_{i=n+1}^{\infty} \sigma_{i}^{2}(A)=\left\|A\left(I-P_{n}\right)\right\|_{\mathrm{HS}}^{2} \leq\left\|A\left(I-Q_{n}\right)\right\|_{\mathrm{HS}}^{2} .
$$

Proof. We shall use the additivity of the singular values, i.e., it holds that

$$
\sigma_{n+i+1}(K+L) \leq \sigma_{n+1}(K)+\sigma_{i+1}(L), \quad \text { for all } n \in \mathbb{N}, i \geq 1 .
$$

In particular we see that

$$
\sigma_{n+i+1}(A) \leq \sigma_{n+1}\left(A Q_{n}\right)+\sigma_{i+1}\left(A\left(I-Q_{n}\right)\right)=\sigma_{i+1}\left(A\left(I-Q_{n}\right)\right),
$$

because $\sigma_{n+1}\left(A Q_{n}\right)$ vanishes by definition of $Q_{n}$. Consequently, we can bound

$$
\begin{aligned}
\sum_{i=n+1}^{\infty} \sigma_{i}^{2}(A) & =\sum_{i=0}^{\infty} \sigma_{n+i+1}^{2}(A) \leq \sum_{i=1}^{\infty} \sigma_{i}^{2}\left(A\left(I-Q_{n}\right)\right) \\
& =\left\|A\left(I-Q_{n}\right)\right\|_{\mathrm{HS}}^{2}
\end{aligned}
$$

with equality for $Q_{n}$ being the singular projections $P_{n}$.

Finally, we mention the following technical result, which is well known. For the sake of completeness, we add a brief proof.

Lemma 5.3. Let $s_{i}(i \in \mathbb{N})$ be non-increasing, and let $\kappa>0$. Suppose that there is a constant $C_{1}<\infty$ such that $\sum_{i=n+1}^{\infty} s_{i}^{2} \leq C n^{-2 \kappa}$ for $n=1,2, \ldots$ Then there is a constant $C_{2}$ such that $s_{i}^{2} \leq C_{2} i^{-(1+2 \kappa)}$ for $i=1,2, \ldots$.

Proof. We can estimate that

$$
n s_{2 n}^{2} \leq \sum_{i=n+1}^{2 n} s_{i}^{2} \leq C n^{-2 \kappa}
$$

which gives $s_{2 n}^{2} \leq C n^{-(1+2 \kappa)}$ and proves the lemma.

Let us introduce the normalized functions

$$
h_{i}(s):=\sqrt{2 i+1} s^{i} \in L^{2}(0,1) \quad(i=0,1,2, \ldots) .
$$

Lemma 5.4. For each $i \geq 1, j \geq 2$, we have that

$$
\left\langle A L_{j}, e_{i}\right\rangle_{\ell^{2}}=-\frac{1}{i \sqrt{2 i+1}}\left\langle h_{i}, L_{j}\right\rangle .
$$


Proof. We have $\left\langle A L_{j}, e_{i}\right\rangle_{\ell^{2}}=\left\langle B^{(\mathrm{H})}\left(J L_{j}\right), e_{i}\right\rangle_{\ell^{2}}$. Using the formula (A.1) with $x:=L_{j}$ $(j=2,3, \ldots)$, and since $L_{j} \perp 1$ for $j \geq 2$, we see that

$$
\left\langle A L_{j}, e_{i}\right\rangle_{\ell^{2}}=-\frac{1}{i}\left[\int_{0}^{1} s^{i} L_{j}(s) \mathrm{d} s\right]=-\frac{1}{i}\left\langle s^{i}, L_{j}\right\rangle_{L^{2}(0,1)}=-\frac{1}{i \sqrt{2 i+1}}\left\langle h_{i}, L_{j}\right\rangle_{L^{2}(0,1)} .
$$

This completes the proof.

Proof of Theorem 5.1. Since the system $\left\{L_{j}\right\}_{j=1}^{\infty}$ of shifted Legendre polynomials is an orthogonal basis in $L^{2}(0,1)$, we have by virtue of [19, Theorem 15.5.5] that

$$
\left\|A\left(I-Q_{n}\right)\right\|_{\mathrm{HS}}^{2}=\sum_{j=1}^{\infty}\left\|A\left(I-Q_{n}\right) L_{j}\right\|_{\ell^{2}}^{2}=\sum_{j=n+1}^{\infty}\left\|A L_{j}\right\|_{\ell^{2}}^{2},
$$

and we shall bound by using Lemma 5.4 that

$$
\begin{aligned}
\left\|A\left(I-Q_{n}\right)\right\|_{\mathrm{HS}}^{2} & =\sum_{i=n}^{\infty} \frac{1}{i^{2}(2 i+1)} \sum_{j=n+1}^{\infty}\left|\left\langle h_{i}, L_{j}\right\rangle_{L^{2}(0,1)}\right|^{2} \\
& =\sum_{i=n}^{\infty} \frac{1}{i^{2}(2 i+1)}\left\|\left(I-Q_{n}\right) h_{i}\right\|_{L^{2}(0,1)}^{2} .
\end{aligned}
$$

The norm square within the above sum is less than or equal to one, so that we arrive at

$$
\left\|A\left(I-Q_{n}\right)\right\|_{\mathrm{HS}}^{2} \leq \sum_{i=n}^{\infty} \frac{1}{i^{2}(2 i+1)} \leq \frac{1}{2} \sum_{i=n}^{\infty} \frac{1}{i^{3}} .
$$

The sum on the right is known to be minus one-half of the second derivative $\psi^{(2)}(n)$ of the digamma function; see [1, (6.4.10)]. Thus we have

$$
\left\|A\left(I-Q_{n}\right)\right\|_{\mathrm{HS}}^{2} \leq \frac{-\psi^{(2)}(n)}{4} .
$$

Moreover, from the series expansion of the digamma function (see [1, (6.4.13)]), we see that $\lim _{n \rightarrow \infty} n^{2} \psi^{(2)}(n)=-1$, which implies that

$$
\left\|A\left(I-Q_{n}\right)\right\|_{\mathrm{HS}}^{2} \leq \frac{1}{3.999 n^{2}}
$$

for sufficiently large $n$. Finally, applying Proposition 5.2 and Lemma 5.3 (with $\kappa=1$ and $s_{i}=\sigma_{i}(A)(i \in \mathbb{N})$ ), we see that $\sigma_{i}(A) \leq C / i^{1.5}$ for some constant $C>0$. This completes the proof.

6. Discussion. We extend the previous discussions in a few aspects. As can be seen from Corollary 3.6 and Theorem 5.1, there is a gap for the composition $B^{(\mathrm{H})} \circ J$ between the obtained decay rate of the order $i^{-3 / 2}$ of the singular values and the available lower bound of the order $\exp (-\underline{C} i)$ as $i \rightarrow \infty$. We shall dwell on this further, and we highlight the main points that are responsible for the lower and upper bounds, respectively.

The overall results are entirely based on considering the Legendre polynomials $L_{j}$ as means for approximation. Clearly, these play a prominent role in our handling of compositions that contain the operator $B^{(\mathrm{H})}$. In particular, the normalized polynomials $L_{j}$ constitute an orthonormal basis in $L^{2}(0,1)$, and the upper bounds from Lemma 3.3 show that these are suited for approximation. However, as a consequence of using the Legendre polynomials, we 
arrive at the $n$-sections of the Hilbert matrix $H_{n}$; see Lemma 3.2. As emphasized in the proof of Theorem 3.1, the condition numbers of the Hilbert matrix $H_{n}$ are of the order $\exp (4 n)$, and this in turn yields the lower bound, after applying Theorem 2.1. Despite the fact that this general result may not be sharp for non-commuting operators in the composition, we may argue that using $n$-sections $H_{n}$ is not a good advice for obtaining sharp lower bounds. So, it may well be that the lower bounds could be improved by using orthonormal bases other than the Legendre polynomials.

The obtained upper bound is based on the approximation of $B^{(\mathrm{H})} \circ J$ by Legendre polynomials in the Hilbert-Schmidt norm, and we refer to the inequality (5.7). There are indications in our analysis, for example, in the context of (5.5), that this bound cannot be improved, but what happens when using other bases?

Another aspect may be interesting. While we established an improved rate for the composition $B^{(\mathrm{H})} \circ J$, this is not possible for the composition $B^{(\mathrm{M})} \circ J$; see the discussion in Section 1. In the light of the spectral theorem, and we omit details, the operator $B^{(\mathrm{M})}$ is orthogonally equivalent to a multiplication operator $M_{f}$ mapping in $L^{2}(0,1)$ with a multiplier function $f$ and possessing zero as accumulation point, and isometries $U: \ell^{2} \rightarrow L^{2}(0,1)$ and $V: L^{2}(0,1) \rightarrow L^{2}(0,1)$, for which we have $B^{(\mathrm{H})}=U^{*} M_{f} V$. This implies that

$$
B^{(\mathrm{H})} \circ J=U^{*} \circ M_{f} \circ V \circ J .
$$

Clearly we have that $\sigma_{i}\left(U^{*} \circ M_{f} \circ V \circ J\right)=\sigma_{i}\left(M_{f} \circ V \circ J\right)$, which looks very similar to the problem of the composition $B^{(\mathrm{M})} \circ J$, where $\sigma_{i}\left(B^{(\mathrm{M})} \circ V \circ J\right) \asymp \sigma_{i}\left(B^{(\mathrm{M})} \circ J\right)$, but with the intermediate isometry $V$. Therefore, we may search for isometries $V: L^{2}(0,1) \rightarrow L^{2}(0,1)$ such that we arrive at $\sigma_{i}\left(B^{(\mathrm{H})} \circ V \circ J\right) \asymp \sigma_{i}\left(B^{(\mathrm{H})} \circ J\right)$. Clearly, this holds true for the identity, and it does not hold true for $V$ from above connected with the Hilbert matrix. Because isometries turn orthonormal bases onto each other, we are again faced with the problem: which approximating orthonormal basis is best suited as a means of approximation in the composition $B^{(\mathrm{H})} \circ J$ ? Thus, the results presented here are only a first step for better understanding the problem of approximating a composition of a compact mapping followed by a non-compact one.

Acknowledgment. The authors express their deep gratitude to Daniel Gerth (TU Chemnitz, Germany) for fruitful discussions and for kindly providing Figure 4.1. We also thank Robert Plato (University of Siegen, Germany) for his hint on studying the Fredholm integral operator kernel of $A^{*} A$ in $L^{2}$, which gives additional motivation. Bernd Hofmann is supported by the German Science Foundation (DFG) under the grant HO 1454/13-1 (Project No. 453804957).

Appendix A. Proof of Proposition 4.2. To prove the series representation (4.3) for the kernel $k$ of $A^{*} A$, we start with

$$
\left[B^{(\mathrm{H})} J x\right]_{j}=\left[\int_{0}^{1}\left(\int_{0}^{t} x(\tau) \mathrm{d} \tau\right) t^{j-1} \mathrm{~d} t\right]_{j} \quad\left(j=1,2, \ldots, x \in L^{2}(0,1)\right) .
$$

Integration by parts yields

$$
\left[B^{(\mathrm{H})} J x\right]_{j}=\left[\frac{1}{j} \int_{0}^{1}\left(1-t^{j}\right) x(t) \mathrm{d} t\right]_{j} \quad(j=1,2, \ldots) .
$$

Taking into account the well-known structure of the adjoint operator $\left(B^{(\mathrm{H})}\right)^{*}$ of $B^{(\mathrm{H})}$ (see, e.g., [7, Proposition 3]), we can further write

$$
\left[\left(B^{(\mathrm{H})}\right)^{*} B^{(\mathrm{H})} J x\right](\eta)=\sum_{j=1}^{\infty} \frac{\eta^{j-1}}{j} \int_{0}^{1}\left(1-t^{j}\right) x(t) \mathrm{d} t \quad(0 \leq \eta \leq 1) .
$$




\section{ETNA}

Kent State University and Johann Radon Institute (RICAM)

Applying the operator $J^{*}$ from the left, for which the analytical structure is also well known, we find again, using integration by parts, the formulas

$$
\begin{aligned}
{\left[A^{*} A x\right](s) } & =\left[J^{*}\left(B^{(\mathrm{H})}\right)^{*} B^{(\mathrm{H})} J x\right](s)=\sum_{j=1}^{\infty} \int_{s}^{1} \frac{\eta^{j-1}}{j}\left(\int_{0}^{1}\left(1-t^{j}\right) x(t) \mathrm{d} t\right) \mathrm{d} \eta \\
& =\sum_{j=1}^{\infty} \frac{1}{j^{2}} \int_{0}^{1}\left(1-t^{j}\right) x(t) \mathrm{d} t-\sum_{j=1}^{\infty} \frac{s^{j}}{j^{2}} \int_{0}^{1}\left(1-t^{j}\right) x(t) \mathrm{d} t \quad(0 \leq s \leq 1) .
\end{aligned}
$$

We can rewrite this as

$$
\left[A^{*} A x\right](s)=\int_{0}^{1}\left(\sum_{j=1}^{\infty} \frac{\left(1-s^{j}\right)\left(1-t^{j}\right)}{j^{2}}\right) x(t) \mathrm{d} t \quad(0 \leq s \leq 1),
$$

which shows the representation (4.3), and this completes the proof.

\section{REFERENCES}

[1] M. Abramowitz And I. A. Stegun, Handbook of Mathematical Functions with Formulas, Graphs, and Mathematical Tables, National Bureau of Standards Applied Mathematics Series No. 55, Washington, D.C., 1964

[2] D. D. Ang, R. Gorenflo, V. K. Le, And D. D. Trong, Moment Theory and Some Inverse Problems in Potential Theory and Heat Condution, Springer, Berlin, 2002.

[3] B. Beckermann, The condition number of real Vandermonde, Krylov and positive definite Hankel matrices, Numer. Math., 85 (2000), pp. 553-577.

[4] R. Boț, B. Hofmann, And P. Mathé, Regularizability of ill-posed problems and the modulus of continuity, Z. Anal. Anwend., 32 (2013), pp. 299-312.

[5] S.-H. Chang, A generalization of a theorem of Hille and Tamarkin with applications, Proc. London Math. Soc. (3), 2 (1952), pp. 22-29.

[6] M. FREITAG AND B. Hofmann, Analytical and numerical studies on the influence of multiplication operators for the ill-posedness of inverse problems, J. Inverse Ill-Posed Probl., 13 (2005), pp. 123-148.

[7] D. Gerth, B. Hofmann, C. Hofmann, And S. Kindermann, The Hausdorff moment problem in the light of ill-posedness of type I, Eurasian J. Math. Comput. Appl., 9 (2021), pp. 57-87.

[8] F. HaUsdorfF, Momentprobleme für ein endliches Intervall, Math. Z., 16 (1923), pp. 220-248.

[9] B. HOFMANN AND S. KINDERMANN, On the degree of ill-posedness for linear problems with non-compact operators, Methods Appl. Anal., 17 (2010), pp. 445-461.

[10] B. Hofmann, P. Mathé, And M. Schieck, Modulus of continuity for conditionally stable ill-posed problems in Hilbert space, J. Inverse Ill-Posed Probl., 16 (2008), pp. 567-585.

[11] B. HOFMANN AND L. VON WOLFERSDORF, Some results and a conjecture on the degree of ill-posedness for integration operators with weights, Inverse Problems, 21 (2005), pp. 427-433.

[12] - A new result on the singular value asymptotics of integration operators with weights, J. Integral Equations Appl., 21 (2009), pp. 281-295.

[13] G. INGLESE, Recent results in the study of the moment problem, in Theory and Practice of Geophysical Data Inversion, A. Vogel, A. K. M. Sarwar, R. Gorenflo,and O. I. Kounchev, eds., Theory and Practice of Applied Geophysics, vol. 5, Vieweg, Wiesbaden, 1992, pp. 73-84.

[14] H. KÖNIG, Eigenvalue Distribution of Compact Operators, Birkhäuser, Basel, 1986.

[15] S. Lu, V. NAUMOVA, AND S. V. PEREVERZEV, Legendre polynomials as a recommended basis for numerical differentiation in the presence of stochastic white noise, J. Inverse Ill-Posed Probl., 21 (2013), pp. $193-216$.

[16] M. Z. NASHED, A new approach to classification and regularization of ill-posed operator equations, in Inverse and Ill-posed Problems, H. W. Engl and C. W. Groetsch, eds., Notes Rep. Math. Sci. Engrg. 4, Academic Press, Boston, 1987, pp. 53-75.

[17] A. PietsCH, s-numbers of operators in Banach spaces, Studia Math., 51 (1974), pp. 201-223.

[18] - Eigenvalues and s-Numbers, Cambridge University Press, Cambridge, 1987.

[19] - Operator Ideals, Dt. Verlag der Wissenschaften, Berlin, 1978.

[20] J. B. READE, Eigenvalues of positive definite kernels, SIAM J. Math. Anal., 14 (1983), pp. 152-157.

[21] - Eigenvalues of Lipschitz kernels, Math. Proc. Cambridge Philos. Soc., 93 (1983), pp. $135-140$.

[22] G. TALENTI, Recovering a function from a finite number of moments, Inverse Problems, 3 (1987), pp. 501-517.

[23] J. TODD, The condition number of the finite segment of the Hilbert matrix, in Contributions to the Solution of Systems of Linear Equations and the Determination of Eigenvalues, O. Taussky, ed., Nat. Bur. of Standards Appl. Math. Series 39, Washington D.C., 1954, pp. 109-116. 
[24] K. T. Vu AND R. Gorenflo, Asymptotics of singular values of fractional integral operators, Inverse Problems, 10 (1994), pp. 949-955.

[25] H. WANG AND S. XIANG, On the convergence rates of Legendre approximation, Math. Comput., 81 (2012), pp. 861-877.

[26] H. S. WILF, Finite Sections of Some Classical Inequalities, Springer, New York, 1970.

[27] Z. ZHAO, A truncated Legendre spectral method for solving numerical differentiation, Int. J. Comput. Math., 87 (2010), pp. 3209-3217. 